\title{
The Information Geometry of the Spherical Model
}

\author{
W. Janke \\ Institut für Theoretische Physik \\ Universität Leipzig \\ Augustusplatz 10/11 \\ D-04109 Leipzig, Germany \\ D.A. Johnston \\ Department of Mathematics \\ Heriot-Watt University \\ Riccarton \\ Edinburgh, EH14 4AS, Scotland \\ and \\ R. Kenna \\ School of Mathematical and Information Sciences \\ Coventry University \\ Coventry, CV1 5FB, England
}

August 14, 2018

\begin{abstract}
Motivated by previous observations that geometrizing statistical mechanics offers an interesting alternative to more standard approaches, we have recently calculated the curvature (the fundamental object in this approach) of the information geometry metric for the Ising model on an ensemble of planar random graphs. The standard critical exponents for this model are $\alpha=-1, \beta=1 / 2, \gamma=2$ and we found that the scalar curvature, $\mathcal{R}$, behaves as $\epsilon^{-2}$, where $\epsilon=\beta_{c}-\beta$ is the distance from criticality. This contrasts with the naively expected $\mathcal{R} \sim \epsilon^{-3}$ and the apparent discrepancy was traced back to the effect of a negative $\alpha$ on the scaling of $\mathcal{R}$.

Oddly, the set of standard critical exponents is shared with the $3 \mathrm{D}$ spherical model. In this paper we calculate the scaling behaviour of $\mathcal{R}$ for the $3 \mathrm{D}$ spherical model, again finding that $\mathcal{R} \sim \epsilon^{-2}$, coinciding with the scaling behaviour of the Ising model on planar random graphs. We also discuss briefly the scaling of $\mathcal{R}$ in higher dimensions, where mean-field behaviour sets in.
\end{abstract}




\section{The Information Geometry of Spin Models}

The idea of endowing the space of parameters with a metric and geometrical structure has been borrowed from parametric statistics [1] and employed to some effect in statistical mechanics [2, 3, 4, 5, 6, 0, 8]. The approach seems to be particularly fruitful for a spin model in field where the parameters are $\beta$, the inverse temperature, and $h$, the external field. In this case the (Fisher-Rao) metric is simply given by

$$
G_{i j}=\partial_{i} \partial_{j} f
$$

where $f$ is the reduced free energy per site and $\partial_{i}=(\partial / \partial \beta, \partial / \partial h)$.

For such a metric the scalar curvature may be calculated as

$$
\mathcal{R}=-\frac{1}{2 G^{2}}\left|\begin{array}{lll}
\partial_{\beta}^{2} f & \partial_{\beta} \partial_{h} f & \partial_{h}^{2} f \\
\partial_{\beta}^{3} f & \partial_{\beta}^{2} \partial_{h} f & \partial_{\beta} \partial_{h}^{2} f \\
\partial_{\beta}^{2} \partial_{h} f & \partial_{\beta} \partial_{h}^{2} f & \partial_{h}^{3} f
\end{array}\right|
$$

where $G=\operatorname{det}\left(G_{i j}\right)$ is the determinant of the metric itself.

The work in [2, 3, 4, 5] has made it clear that, as one might expect, the scalar curvature plays a central role in any attempt to look at statistical mechanics from a geometrical perspective. In particular, for all the models that have been considered so far, the curvature diverges only at a phase transition point for physical ranges of the parameter values. We assume a standard scaling form for the free energy per site

$$
f(\epsilon, h)=\lambda^{-1} f\left(\epsilon \lambda^{a_{\epsilon}}, h \lambda^{a_{h}}\right),
$$

where $\epsilon=\beta_{c}-\beta$ (with $\beta_{c}$ marking the critical point) and $a_{\epsilon}, a_{h}$ are the scaling dimensions for the energy and spin operators.

The spherical model has a one-sided critical point [9] and we are interested in the high-temperature domain (where $\epsilon>0$ ). There, standard scaling assumptions allow us to write (3) as

$$
f(\epsilon, h)=\epsilon^{1 / a_{\epsilon}} \psi_{+}\left(h \epsilon^{-a_{h} / a_{\epsilon}}\right),
$$

where we have introduced the scaling function $\psi_{+}$. Consideration of the behaviour of the components of $\mathcal{R}$ in equ. (2) then leads to the scaling

$$
\mathcal{R} \sim \epsilon^{\alpha-2}
$$

for the curvature itself.

Equation (5) shows that the scalar curvature not only characterises phase transition points as divergences, as do the more standard statistical mechanical quantities such as the specific heat $C$ and susceptibility $\chi$, it displays a scaling behaviour that allows the extraction of the critical exponent $\alpha$. Indeed, with hyperscaling, equ. (5) can be recast as

$$
\mathcal{R} \sim \xi^{d}
$$

where $\xi$ is the correlation length and $d$ is the dimensionality of the system. So the curvature is proportional to the correlation volume, an intuitively reasonable result on dimensional grounds. 
At first sight, our calculation of the scaling behaviour of $\mathcal{R}$ in [10] for the Ising model on planar random graphs puts a spanner in the works. There, $\alpha=-1$ but $\mathcal{R} \sim \epsilon^{-2}$ rather than the expected $\mathcal{R} \sim \epsilon^{-3}$. However, returning to the detailed scaling of the individual terms in equ. (2) showed that a negative $\alpha$ affected some of the scaling of the components and the end result could be traced back to these modifications. In principle this modified behaviour should apply to any model with negative $\alpha$, so an interesting test would be to calculate $\mathcal{R}$, or at least its scaling limit, for another model with this property.

The spherical model provides just such a test case. It was solved (in field) in the classic Berlin and Kac paper [9] and the critical exponents in 3D found to be $\alpha=-1, \beta=$ $1 / 2, \gamma=2$ which are identical to those of the Ising model on 2D planar random graphs [11]. This is remarkable, because there are no obvious physical similarities between the two models. An additional motivation for any such calculation is the general paucity of spin models that have been solved in field. Indeed, $\mathcal{R}$ for spin models has been obtained explicitly only for the 1D Ising [3], Bethe lattice Ising [6] and 1D Potts [12] models and the scaling form calculated for the Ising model on planar random graphs 10. This forms a rather small sample on which to formulate hypotheses about its general behaviour and its scaling properties. In the sequel, we extend this short list incrementally by looking at the scaling behaviour of $\mathcal{R}$ in the spherical model. We concentrate on the 3D case, but also discuss other dimensions, including the mean-field like behaviour which sets in at $d=4$.

\section{The Spherical Model}

Berlin and Kac [9] introduced the spherical model (and the Gaussian model) in an attempt to understand how generic some of the features of Onsager's solution [13] of the 2D Ising model are for ferromagnetic spin models, particularly for other dimensions. In the spherical model, the \pm 1 condition on the value of the Ising spins is relaxed, whilst retaining a global constraint on the total spin magnitude. With $s_{i}$ denoting the value of a spin at a site $i$ of a hypercubic lattice, the partition function is [9]

$$
\mathcal{Z}=\int d s_{1} \ldots d s_{N} \exp \left(\beta \sum_{\langle i j\rangle} s_{i} s_{j}+h \sum_{i} s_{i}\right) \delta\left(\sum_{i} s_{i}^{2}-N\right),
$$

where $N$ is the total number of sites. This can be evaluated by exponentiating the constraint and using steepest descent, resulting in the following expression for the reduced free energy per site in the thermodynamic limit, $N \rightarrow \infty$ :

$$
f=\frac{1}{2} \log \left(\frac{\pi}{\beta}\right)+\beta z-\frac{1}{2} g(z)+\frac{h^{2}}{4 \beta(z-d)},
$$

where

$$
g(z)=\frac{1}{(2 \pi)^{d}} \int_{0}^{2 \pi} d \omega_{1} \ldots d \omega_{d} \log \left(z-\sum_{k=1}^{d} \cos \left(\omega_{k}\right)\right) .
$$

The saddle point value of $z$ which appears in the expression for the free energy in equ. (8) is determined from

$$
g^{\prime}(z)=2 \beta-\frac{h^{2}}{2 \beta(z-d)^{2}}
$$


The solution reveals no transition for $d=1$ and 2 , and a transition with the exponents $\alpha=-1, \beta=1 / 2, \gamma=2$ for $d=3$. For $d \geq 4$, on the other hand, mean-field behaviour with $\alpha=0, \beta=1 / 2, \gamma=1$ sets in (modified by multiplicative logarithmic corrections in the $d=4$ case $[14]$ ). For the following formulae we confine our attention to the $d=3$ case.

It is useful to note that, with $h=0$, equ. (10) gives

$$
\frac{d z}{d \beta}=\frac{2}{g^{\prime \prime}(z)}
$$

and hence

$$
\frac{d^{2} z}{d \beta^{2}}=-\frac{4 g^{(3)}(z)}{\left[g^{\prime \prime}(z)\right]^{3}}
$$

The critical point is given by $z=d=3$ and $h=0$ [9], and the behaviour of $g(z)$ in this region is determined by differentiating equ. (9) twice and then expanding for the small $\omega_{k}$ values which give the dominant contribution. One finds

$$
g^{\prime \prime}(z) \sim-\frac{1}{2 \sqrt{2} \pi}(z-3)^{-1 / 2} .
$$

A further differentiation gives

$$
g^{(3)}(z) \sim \frac{1}{4 \sqrt{2} \pi}(z-3)^{-3 / 2}
$$

and an integration yields

$$
g^{\prime}(z)=\frac{1}{\sqrt{2} \pi}(z-3)^{1 / 2}+g^{\prime}(3)
$$

where $g^{\prime}(3)=(18+12 \sqrt{2}-10 \sqrt{3}-7 \sqrt{6})[K(2 \sqrt{3}+\sqrt{6}-2 \sqrt{2}-3)]^{2} \approx 0.505462019 \ldots$ is the exactly known massless $3 \mathrm{D}$ lattice propagator at the origin, with $K\left(k^{2}\right)$ denoting the standard elliptic integral. This latter expression can be combined with equ. (10) with $h=0$ to give

$$
(z-3) \sim 8 \pi^{2}\left(\beta_{c}-\beta\right)^{2} \sim \epsilon^{2},
$$

in which $\beta_{c}=g^{\prime}(3) / 2 \approx 0.252731009 \ldots$ Equations (13) and (14) may then be substituted in equs. (11) and (12) to give the scaling of $d z / d \beta$ and $d^{2} z / d \beta^{2}$,

$$
\begin{aligned}
\lim _{z \rightarrow 3} \frac{d z}{d \beta} & =\lim _{z \rightarrow 3}\left\{-4 \sqrt{2} \pi(z-3)^{1 / 2}\right\}=0, \\
\lim _{z \rightarrow 3} \frac{d^{2} z}{d \beta^{2}} & =16 \pi^{2},
\end{aligned}
$$

which we shall employ below in the calculation of the scalar curvature. 


\section{The Scalar Curvature}

We recapitulate the general considerations of [10] before going on to discuss in detail the results for the spherical model. In (更), we define $A=1 / a_{\epsilon}$ and $C=-a_{h} / a_{\epsilon}$, which, in terms of the standard exponents, gives $A=2-\alpha$ and $A+C=\beta$. If $A>2$ (which is the case for $\alpha<0$ ) the specific heat, rather than diverging, will be a constant at the critical point, which we denote by $A(A-1) \phi(0)$. With this in mind, substitution of the scaling function into equ. (2) gives

$$
\begin{aligned}
& \mathcal{R}=-\frac{1}{2 G^{2}} \times \\
& A(A-1) \phi(0) \\
& \begin{array}{ccc}
A(A-1)(A-2) \epsilon^{A-3} \psi_{+}(0) & 0 & \epsilon^{A+2 C} \psi_{+}^{\prime \prime}(0) \\
0 & -(A+2 C) \epsilon^{A+2 C-1} \psi_{+}^{\prime \prime}(0) & -(A+2 C) \epsilon^{A+2 C-1} \psi_{+}^{\prime \prime}(0)
\end{array} \mid .
\end{aligned}
$$

In equ. (18) terms with an odd number of $h$ derivatives have been set to zero. These

do not appear because of the $h \rightarrow-h$ symmetry of the free energy. The scaling for the determinant of the metric $G$ is given by

$$
G=A(A-1) \epsilon^{A+2 C} \phi(0) \psi_{+}^{\prime \prime}(0) \quad,
$$

so the leading term in $\mathcal{R}$ (for $A>2$ ) is

$$
\mathcal{R}=\frac{(A+2 C)^{2}}{2 A(A-1) \phi(0)} \epsilon^{-2},
$$

or, translating back to the standard critical exponents,

$$
\mathcal{R}=\frac{\gamma^{2}}{2(2-\alpha)(1-\alpha) \phi(0)} \epsilon^{-2} .
$$

In summary, if $\alpha<0$ the expected scaling of $\mathcal{R}$ is $\mathcal{R} \sim \epsilon^{-2}$ rather than the $\mathcal{R} \sim \epsilon^{\alpha-2}$, which is seen for positive $\alpha$.

We now move on to examine the scaling of the various terms contributing to $\mathcal{R}$ in equ. (2) for the spherical model itself. As we have remarked, the $h \rightarrow-h$ symmetry in the free energy per site, $f$, of the spherical model means that any terms with an odd number of $h$ derivatives will automatically be zero when $h=0$, hence $f_{\beta h}=f_{\beta \beta h}=f_{h h h}=0$. This leaves the non-zero terms (again when $h=0$ )

$$
\begin{aligned}
f_{\beta \beta} & =\frac{\partial z}{\partial \beta}+\frac{1}{2 \beta^{2}}, \\
f_{h h} & =\frac{1}{2 \beta(z-3)}, \\
f_{\beta \beta \beta} & =\frac{\partial^{2} z}{\partial \beta^{2}}-\frac{1}{\beta^{3}}, \\
f_{h h \beta} & =-\frac{1}{2 \beta(z-3)^{2}} \frac{\partial z}{\partial \beta}-\frac{1}{2 \beta^{2}(z-3)},
\end{aligned}
$$


which, using equs. (16) and (17) have the following behaviour in the scaling region:

$$
\begin{aligned}
f_{\beta \beta} & \sim \frac{1}{2 \beta_{c}^{2}}, \\
f_{h h} & \sim \frac{1}{16 \pi^{2} \beta_{c}\left(\beta_{c}-\beta\right)^{2}} \sim \epsilon^{-2}, \\
f_{\beta \beta \beta} & \sim 16 \pi^{2}-\frac{1}{\beta_{c}^{3}}, \\
f_{h h \beta} & \sim \frac{1}{8 \pi^{2} \beta_{c}\left(\beta_{c}-\beta\right)^{3}}-\frac{1}{16 \pi^{2} \beta_{c}^{2}\left(\beta_{c}-\beta\right)^{2}} \sim \epsilon^{-3} .
\end{aligned}
$$

Comparing with the individual terms in equ. (18) we see that the expected general scaling of each term (for $\alpha<0$ ) does indeed apply and that overall we have, as in equ. (21),

$$
\mathcal{R} \sim \epsilon^{-2}
$$

We thus see that calculating the scaling of $\mathcal{R}$ for the $3 \mathrm{D}$ spherical model for which $\alpha=-1$ gives results in accordance with expectations from general scaling arguments which take into account the negative $\alpha$, similarly to the Ising model on planar random graphs.

In $d \geq 4$ dimensions the exponents of the spherical model attain their mean-field values, $\alpha=0, \beta=1 / 2, \gamma=1$. In this case $g^{\prime}(z) \sim z-d+g^{\prime}(d)$ near criticality and the resulting scaling of the components of the expression for $\mathcal{R}$ is

$$
\mathcal{R}=-\frac{1}{2 G^{2}}\left|\begin{array}{ccc}
\text { const } & 0 & \epsilon^{-1} \\
\text { const } & 0 & \epsilon^{-2} \\
0 & \epsilon^{-2} & 0
\end{array}\right|
$$

where the determinant of the metric scales as $G=\epsilon^{-1}$. Thus $\mathcal{R}$ itself scales as $\epsilon^{-2}$, in the mean-field case. This is in agreement with the expected scaling of $\epsilon^{\alpha-2}$ when $\alpha \geq 0$ since, in that case [4],

$$
\begin{aligned}
& \mathcal{R}=-\frac{1}{2 G^{2}} \times \\
& \left|\begin{array}{ccc}
A(A-1) \epsilon^{A-2} \psi_{+}(0) & 0 & \epsilon^{A+2 C} \psi_{+}^{\prime \prime}(0) \\
-A(A-1)(A-2) \epsilon^{A-3} \psi_{+}(0) & 0 & -(A+2 C) \epsilon^{A+2 C-1} \psi_{+}^{\prime \prime}(0) \\
0 & -(A+2 C) \epsilon^{A+2 C-1} \psi_{+}^{\prime \prime}(0) & 0
\end{array}\right|
\end{aligned}
$$

where the scaling of the metric determinant is

$$
G=A(A-1) \epsilon^{2 A+2 C-2} \psi_{+}(0) \psi_{+}^{\prime \prime}(0)
$$

The difference in scaling between the $\alpha \geq 0$ and the $\alpha<0$ cases originates in the $f_{\beta \beta}$ term which contributes to both $\mathcal{R}$ and $G$ in equ. (26) and equ. (27). In the negative $\alpha$ case, the contribution of this term comes from the regular part of the free energy, and is non-diverging.

Note that for $\alpha=0, A=2$, and although the displayed scaling term $A(A-1)(A-$ 2) $\epsilon^{A-3} \psi_{+}(0)$ in equ. (26) would be expected to vanish, a regular term could still contribute. 
This is indeed what is seen in the explicit calculation for the spherical model. Two further caveats arise. Firstly, for the upper critical dimension $d=4$ itself, there are multiplicative logarithmic corrections [14] which require a careful handling. Secondly, for $d>4$, where the scaling is described by mean-field theory, the hyperscaling relation fails. So one should be quite cautious in comparing the explicit calculation in terms of $\epsilon$ with expressions involving the correlation length $\xi$.

\section{Conclusions}

We have calculated the scaling behaviour of the scalar curvature, $\mathcal{R}$, of the information geometry metric for the 3D spherical model, finding, as for the Ising model on planar random graphs, that $\mathcal{R} \sim \epsilon^{-2}$. Careful considerations of the scaling of the various elements which contribute to $\mathcal{R}$ show that this is in accordance with expectations. We also briefly discussed the scaling behaviour of $\mathcal{R}$ for $d \geq 4$, corresponding to mean-field exponents.

The calculation reported here provides another example of a statistical mechanical model in which the curvature of the information geometry metric diverges at the critical point, with a clearly quantifiable scaling behaviour. Once again it is curious that the relevant exponent is shared with the Ising model on planar random graphs. All critical exponents now coincide in the two models though there is no obvious physical relation between them. It seems that the suggestion in the original Berlin and Kac paper 9] that the 3D spherical model might provide a hint to the behaviour of the 3D Ising model applies rather to the Ising model on 2D planar random graphs.

\section{Acknowledgements}

W.J. and D.J. were partially supported by EC IHP network "Discrete Random Geometries: From Solid State Physics to Quantum Gravity" HPRN-CT-1999-000161. D.J. and R.K. were also partially supported by an Enterprise Ireland/British Council Research Visits Scheme grant.

\section{References}

[1] R.A. Fisher, Phil. Trans. R. Soc. Lond. 222 (1922) 309;

C.R. Rao, Bull. Calcutta Math. Soc. 37 (1945) 81.

[2] G. Ruppeiner, Rev. Mod. Phys. 67 (1995) 605; Phys. Rev. A 20 (1979) 1608; ibid. A 24 (1980) 488.

[3] H. Janyszek, Rep. Math. Phys. 24 (1986) 1; ibid. 11;

H. Janyszek and R. Mrugała, Phys. Rev. A 39 (1989) 6515.

[4] H. Janyszek, J. Phys. A 23 (1990) 477.

[5] E.J. Brody, Phys. Rev. Lett. 58 (1987) 179;

D. Brody and N. Rivier, Phys. Rev. E 51 (1995) 1006; 
D. Brody and A. Ritz, Nucl. Phys. B 522 (1998) 588;

D. Brody and L. Hughston, Proc. Roy. Soc. London A 455 (1999) 1683.

[6] B.P. Dolan, Proc. Roy. Soc. London A 454 (1998) 2655.

[7] B.P. Dolan, Int. J. Mod. Phys. A 12 (1997) 2413.

[8] D. O'Connor and C.R. Stephens, Prog. Theor. Phys. 90 (1993) 747;

B.P. Dolan, Int. J. Mod. Phys. A 10 (1995) 2439; ibid. 2703; Nucl. Phys. B 528 (1998) 553;

J. Gaite and D. O'Connor, Phys. Rev. D 54 (1996) 5163;

A.C. Irving and J.C. Sexton, Phys. Rev. D 55 (1997) 5456.

[9] T. Berlin and M. Kac, Phys. Rev. 86 (1952) 821.

[10] W. Janke, D.A. Johnston, and Ranasinghe P.K.C. Malmini, "The Information Geometry of the Ising Model on Planar Random Graphs", preprint cond-mat/0207573, to appear in Phys. Rev. E.

[11] V.A. Kazakov, Phys. Lett. A 119 (1986) 140;

D.V. Boulatov and V.A. Kazakov, Phys. Lett. B 186 (1987) 379.

[12] B.P. Dolan, D.A. Johnston, and R. Kenna, J. Phys. A 35 (2002) 9025.

[13] L. Onsager, Phys. Rev. 65 (1944) 117.

[14] R. Kenna and C.B. Lang, Phys. Lett. B 264 (1991) 396; Nucl. Phys. B 393 (1993) 461. 